\title{
EXTREMAL MANIFOLDS AND HAUSDORFF DIMENSION
}

\section{H. DICKINSON AND M. M. DODSON}

1. Introduction. The recent proof by D. Y. Kleinbock and G. A. Margulis [11] of Sprindžuk's conjecture for smooth nondegenerate manifolds $M$ means that the set $\mathscr{L}_{v}(M)$ of $v$-approximable points (this and other terminology is explained below) on $M$ is of zero induced Lebesgue measure. This raises the question of its Hausdorff dimension. Bounds and indeed the exact dimension for manifolds satisfying a variety of arithmetic, geometric, and analytic conditions are known (see [2], [3], [5], [7]). In this paper ubiquity is used to obtain a lower bound for the Hausdorff dimension of a set more general than $\mathscr{L}_{v}(M)$ for any extremal $C^{1}$ manifold $M$. Hitherto volume estimates that depend on curvature conditions were used to overcome a "small denominators" problem. It turns out, however, that extremality, when combined with Fatou's lemma, is all that is needed. We begin with some notation.

Let $|x|=\max \left\{\left|x_{1}\right|, \ldots,\left|x_{n}\right|\right\}$ denote the supremum norm or height of the point $x=\left(x_{1}, \ldots, x_{n}\right)$ in $n$-dimensional Euclidean space $\mathbb{R}^{n}$, and denote its Euclidean norm by $|x|_{2}=\left(x_{1}^{2}+\cdots+x_{n}^{2}\right)^{1 / 2}$. Throughout, $\mathbf{q}=\left(q_{1}, \ldots, q_{n}\right)$ is a vector in $\mathbb{Z}^{n}$, and $\mathbf{q} \cdot x=q_{1} x_{1}+\cdots+q_{n} x_{n}$ denotes the usual inner product. For positive numbers $a, b$, we use the Vinogradov notation $a \ll b$ and $b \gg a$ if $a=O(b)$. If $a \ll b \ll a$, we write $a \asymp b$. A point $x \in \mathbb{R}^{n}$ that satisfies

$$
\|\mathbf{q} \cdot x\|<|\mathbf{q}|^{-v}
$$

for infinitely many $\mathbf{q} \in \mathbb{Z}^{n}$ is called $v$-approximable ( $\|x\|$ is the distance of the real number $x$ from $\mathbb{Z}$ ). Let $M$ be an $m$-dimensional manifold in $\mathbb{R}^{n}$. The set of $v$-approximable points in the manifold $M$ is denoted by $\mathscr{L}_{v}(M)$. The manifold $M$ is called extremal if for any $v>n, \mathscr{L}_{v}(M)$ has Lebesgue measure 0 . Equivalently, by Khintchine's transference principle, $M$ is extremal if the set $\mathscr{S}_{w}(M)$ of points $x \in M$ that are simultaneously $w$-approximable (i.e., for which

$$
\|q x\|<|q|^{-w}
$$

for infinitely many $q \in \mathbb{Z}$ ) is null (i.e., of measure zero) when $w>1 / n$. Khintchine's theorem implies that the real line is extremal, and the terminology reflects the fact that

Received 24 November 1998.

1991 Mathematics Subject Classification. Primary 11J83; Secondary 11J20.

Dickinson supported by Engineering and Physical Sciences Research Council grant number GR/ K56407. 
the order of approximation given by Dirichlet's theorem is unimprovable for almost all points on an extremal manifold (see [12]).

Let $U$ be an open set in $\mathbb{R}^{m}$, where $m \leqslant n$. V. G. Sprindžuk conjectured that if the functions $\theta_{j}: U \rightarrow \mathbb{R}, j=1, \ldots, n$ are analytic and, together with 1 , independent over $\mathbb{R}$, then the manifold

$$
\left\{\left(\theta_{1}(u), \ldots, \theta_{n}(u)\right): u \in U\right\}=\theta(U) \subset \mathbb{R}^{n}
$$

is extremal (see Conjecture $\mathrm{H}_{1}$ in [19]). Manifolds satisfying a variety of additional or different analytic, geometric, and number-theoretic conditions have been shown to be extremal; references and further details can be found in [18], [19] (see also [4], [7], [9], [11], [20]).

In the stronger Baker-Sprindžuk conjecture, the hypotheses on the manifold $M$ are the same, but the approximation function $|\mathbf{q}|^{-v}$ is replaced by a larger multiplicative anisotropic function. When $v>n$, if the set of points $x \in M$ for which

$$
\|\mathbf{q} \cdot x\|<\prod_{j=1}^{n}\left(\left|q_{j}\right|+1\right)^{-v / n}
$$

for infinitely many $\mathbf{q} \in \mathbb{Z}^{n}$ is relatively null, then $M$ is said to be strongly extremal (see Conjecture $\mathrm{H}_{2}$ in [19]). Points satisfying (2) for infinitely many $\mathbf{q} \in \mathbb{Z}^{n}$ are called multiplicatively $v$-approximable. Transference principles allow simultaneous and multiplicative approximation forms of these conjectures (see [11], [18]). The conjecture $\mathrm{H}_{2}$ was first proposed by A. Baker for the rational normal curve

$$
\mathscr{V}=\left\{\left(t, t^{2}, \ldots, t^{n}\right): t \in \mathbb{R}\right\}
$$

in [1] and proved for this case by V. I. Bernik [6].

J. Kubilius proved the parabola extremal in 1949 [13], and in 1964 W. M. Schmidt established the remarkable result that any $C^{3}$ planar curve with nonzero curvature almost everywhere is extremal [16]. About the same time, Sprindžuk proved Mahler's conjecture, corresponding to the rational normal curve being extremal (see [17]). Recently, in [11], Kleinbock and Margulis have proved a result that implies not only Sprindžuk's conjecture $\mathrm{H}_{1}$, but also the Baker-Sprindžuk conjecture $\mathrm{H}_{2}$. They used ideas from dynamical systems, namely, unipotent flows in homogeneous spaces of lattices and the correspondence between multiplicatively $v$-approximable points for $v>n$ and unbounded orbits in the space of lattices. Although at the moment their techniques do not yield nontrivial upper bounds for the Hausdorff dimension, they do give a partial Khintchine-type result and might open the way to further progress.

In [3], R. C. Baker refined Schmidt's result [16] by showing that if the curvature of a $C^{3}$ planar curve vanishes only on a set with Hausdorff dimension 0 , then for $v \geqslant 2$,

$$
\operatorname{dim} \mathscr{L}_{v}(M)=\frac{3}{v+1} .
$$

Using the idea of regular systems, A. Baker and Schmidt [2] showed that $\operatorname{dim} \mathscr{L}_{v}(\mathscr{V}) \geqslant$ 
$(n+1) /(v+1)$ for $v \geqslant n$. The complementary upper inequality was established by Bernik [5], giving

$$
\operatorname{dim} \mathscr{L}_{v}(\mathscr{V})=\frac{n+1}{v+1}
$$

for $v \geqslant n$. For manifolds $M$ with dimension $m \geqslant 2$ and satisfying a curvature condition that reduces to nonvanishing Gaussian curvature for surfaces in $\mathbb{R}^{3}$,

$$
\operatorname{dim} \mathscr{L}_{v}(M)=m-1+\frac{n+1}{v+1}
$$

for $v \geqslant n$ (see [7]). We use ubiquity (see [8]) to obtain the best possible lower bound for the Hausdorff dimension of the more general set

$$
\mathscr{L}(M ; \psi)=\left\{x \in M:\|\mathbf{q} \cdot x\|<\psi(|\mathbf{q}|) \text { for infinitely many } \mathbf{q} \in \mathbb{Z}^{n}\right\}
$$

when $M$ is a $C^{1}$ extremal manifold in $\mathbb{R}^{n}$ and the function $\psi: \mathbb{N} \rightarrow \mathbb{R}^{+}$decreases. Note that when $\psi(q)=q^{-v}$, we write $\mathscr{L}_{v}(M)$ for $\mathscr{L}(M ; \psi)$. For more information about Hausdorff dimension, see [10], [14].

Acknowledgements. We are grateful to Dmitry Kleinbock and Gregor Margulis for helpful and stimulating discussions during the Dynamical Systems and Related Topics meeting held in 1997 at the Erwin Schrödinger Institute for Mathematical Physics in Vienna, to Chris Wood for his help with the differential geometry, and to Alan Baker and Bryan Rynne for their comments on earlier drafts. We are also grateful to the referee for suggesting ways of improving our presentation. We would like to thank the Engineering and Physical Sciences Research Council for enabling us to participate in the Vienna meeting.

2. Ubiquitous systems. Let $U$ be a nonempty open subset of $\mathbb{R}^{m}$. Let

$$
\mathscr{R}=\left\{R_{j} \subset U: j \in J\right\}
$$

be a family of sets indexed by $J$; these sets are called resonant. Suppose further that each $j \in J$ has a weight $\lfloor j\rfloor>0$, and let $\rho: \mathbb{N} \rightarrow \mathbb{R}^{+}$be a function converging to zero at infinity. Suppose that for each sufficiently large positive integer $N$, there exists a set $A(N) \subset U$ for which

$$
\lim _{N \rightarrow \infty}|U \backslash A(N)|=0 .
$$

Let

$$
B\left(R_{j} ; \delta\right)=\left\{u \in U: \operatorname{dist}\left(u, R_{j}\right)<\delta\right\},
$$

where $\operatorname{dist}(u, R)=\inf \{|u-r|: r \in R\}$. Let $H / 2$ denote the hypercube $H$ shrunk by $1 / 2$ and with the same centre. 
Suppose that there exists a constant $d \in[0, m]$ such that given any hypercube $H \subset U$ with sidelength $\ell(H)=\rho(N)$ and $H / 2$ meeting $A(N)$, there exists a $j \in J$ with $\lfloor j\rfloor \leqslant N$ such that for all $\delta \in(0, \rho(N)]$,

$$
\left|H \cap B\left(R_{j} ; \delta\right)\right| \gg \delta^{m-d} \ell(H)^{d} .
$$

Suppose further that given any other hypercube $H^{\prime}$ in $U$ with $\ell\left(H^{\prime}\right) \leqslant \rho(N)$,

$$
\left|H^{\prime} \cap H \cap B\left(R_{j} ; \delta\right)\right| \ll \delta^{m-d} \ell\left(H^{\prime}\right)^{d} .
$$

Then the pair $(\mathscr{R},\lfloor\cdot\rfloor)$ is called a ubiquitous system with respect to $\rho$.

In the one-dimensional case and when the resonant sets consist of points, ubiquitous and regular systems are virtually equivalent and essentially differ only in their formulation (see [15]).

3. Hausdorff dimension. The distribution of the resonant sets in ubiquitous systems allows the determination of a general lower bound for the lim-sup set

$$
\Lambda(\mathscr{R} ; \psi)=\left\{u \in U: \operatorname{dist}\left(u, R_{j}\right)<\psi(\lfloor j\rfloor) \text { for infinitely many } j \in J\right\},
$$

where $\psi: \mathbb{N} \rightarrow \mathbb{R}^{+}$is a decreasing function (see [8]).

Theorem 1. Suppose $(\mathscr{R},\lfloor\cdot\rfloor)$ is ubiquitous with respect to $\rho: \mathbb{N} \rightarrow \mathbb{R}^{+}$and that $\psi: \mathbb{N} \rightarrow \mathbb{R}^{+}$is a decreasing function satisfying $\psi(N) \leqslant \rho(N)$ for $N$ sufficiently large. Then

$$
\operatorname{dim} \Lambda(\mathscr{R} ; \psi) \geqslant d+\gamma(m-d)
$$

where $\gamma=\lim \sup _{N \rightarrow \infty}(\log \rho(N)) /(\log \psi(N)) \leqslant 1$.

The hypothesis that $\psi(N) \leqslant \rho(N)$ for $N$ sufficiently large implies that $\gamma \leqslant 1$. We now apply Theorem 1 to Diophantine approximation on a manifold. The lower order $\lambda(f)$ of the function $f: \mathbb{N} \rightarrow \mathbb{R}^{+}$is defined by

$$
\lambda(f)=\liminf _{N \rightarrow \infty} \frac{\log f(N)}{\log N} .
$$

THEOREM 2. Let $M$ be an m-dimensional $C^{1}$ extremal manifold embedded in $\mathbb{R}^{n}$. Let $\psi: \mathbb{N} \rightarrow \mathbb{R}^{+}$be decreasing with the lower order of $1 / \psi$ denoted by $\lambda$. Then for $\lambda \geqslant n$,

$$
\operatorname{dim} \mathscr{L}(M ; \psi) \geqslant m-1+\frac{n+1}{\lambda+1} .
$$

Since $\operatorname{dim} \mathscr{L}(M \cap V ; \psi) \leqslant \operatorname{dim} \mathscr{L}(M ; \psi)$, it suffices to consider the open subset $M \cap V$ of $M$, where $V$ is a suitable open set in $\mathbb{R}^{n}$. We assume without loss of 
generality that $M \cap V \subset[-1,1]^{n}$ and that $V$ is sufficiently small. Let $\theta: U \rightarrow M \cap V$ be the local parametrisation where the domain $U$ is a sufficiently small hypercube in $[-1,1]^{m}$. We write $M_{U}=M \cap V=\theta(U)$. Each point $x \in M_{U}$ can be written $x=\theta(u)$ for some $u \in U$.

Since the manifold $M$ is $C^{1}$, by shrinking and closing $U$ if necessary, we can assume that the geodesic distance between two points $x, x^{\prime}$ on $M_{U}$ is comparable with $\left|x-x^{\prime}\right|$ and that $\theta$ is bi-Lipschitz on $U$. Hence we can assume that the Hausdorff dimension of $\mathscr{L}\left(M_{U} ; \psi\right)$ and that of

$$
L(\psi)=\left\{u \in U:\|\mathbf{q} \cdot \theta(u)\|<\psi(|\mathbf{q}|) \text { for infinitely many } \mathbf{q} \in \mathbb{Z}^{n}\right\}
$$

are the same (see [10]). We write $L_{v}$ for $L(\psi)$ when $\psi(r)=r^{-v}$; thus

$$
L_{v}=\left\{u \in U:\|\mathbf{q} \cdot \theta(u)\|<|\mathbf{q}|^{-v} \text { for infinitely many } \mathbf{q} \in \mathbb{Z}^{n}\right\} .
$$

By the inverse function theorem, we can also assume that $M_{U}$ is the graph of a $C^{1}$ (Monge) ordinate function $\varphi: U \rightarrow \mathbb{R}^{k}$, where $k=n-m$, so that

$$
M_{U}=\{\theta(u): u \in U\}=\{(u, \varphi(u)): u \in U\}
$$

and $\theta=1_{U} \times \varphi$. The corresponding local chart $h: M_{U} \rightarrow U$ is the restriction to $M_{U}$ of the projection $\mathbb{R}^{m} \times \mathbb{R}^{k} \rightarrow \mathbb{R}^{m}$. Moreover, by shrinking and closing $U$ again if necessary, we can assume $\left|\partial \varphi_{j} / \partial u_{i}\right| \leqslant K_{i j}<\infty$ for each $u \in U, i=1, \ldots, m$, $j=1, \ldots, k$. Indeed given $\delta>0$, we can choose $U$ so that for any $u \in U$,

$$
K_{i j}-\delta \leqslant\left|\frac{\partial \varphi_{j}(u)}{\partial u_{i}}\right| \leqslant K_{i j} .
$$

Thus we can assume that the change in the direction of a vector along any geodesic in $M_{U}$ is small.

It follows that $M_{U}$ is not close to orthogonal to $\mathbb{R}^{m} \times\{0\}, 0=(0, \ldots, 0) \in \mathbb{R}^{k}$, as indicated in Figure 1. More precisely, for each $\theta(u)$ in $M_{U}$, the angle $\vartheta$, say, between any vector in the tangent space $T_{\theta(u)} M_{U}$ and $\mathbb{R}^{m} \times\{0\}$, satisfies $\cos \vartheta \geqslant c$ for some constant $c>0$ (i.e., in the Vinogradov notation, $\cos \vartheta \gg 1$ ). Thus for any $\theta(u)$ in $M_{U}$, the plane $\mathbb{R}^{m} \times\{0\}$ is not close to being orthogonal to $T_{\theta(u)} M_{U}$. In other words, the normal space $T_{\theta(u)} M_{U}^{\perp}$ is not close to being parallel to $\mathbb{R}^{m} \times\{0\}$.

Since $M$ is extremal, $\mathscr{L}_{v}\left(M_{U}\right)=\theta\left(L_{v}\right)$ is null for $v>n$ in the induced measure on $M$ and, since $\theta$ is bi-Lipschitz on $U$, the set $L_{v}$ is null in $\mathbb{R}^{m}$ when $v>n$. To obtain a lower bound for the Hausdorff dimension of $\mathscr{L}_{v}(M)$ or equivalently for $L_{v}$, it suffices to find a sequence of suitable sets $A(N) \subset U$ that approximate $U$ in measure and that satisfy the intersection conditions (5) and (6) above. Using the geometry of numbers, integer vectors $\mathbf{q}$ are chosen so that the hyperplanes

$$
\Pi_{p, \mathbf{q}}=\left\{x \in \mathbb{R}^{n}: \mathbf{q} \cdot x=p\right\}
$$




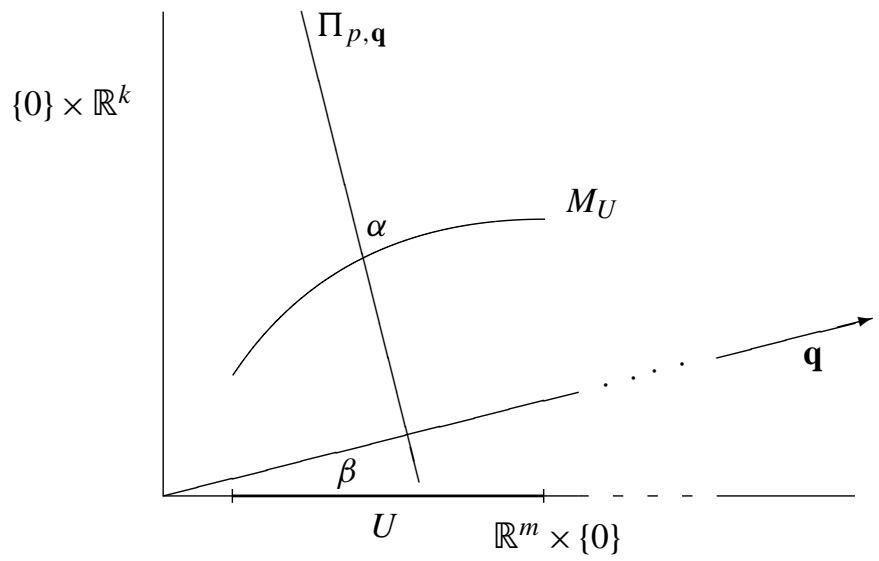

Figure 1. The manifold $M_{U}$ and a resonant set $\Pi_{p, \mathbf{q}}$

associated with the resonant sets $R_{p, \mathbf{q}}$, defined below in (11), are not close to being parallel or tangential to $M_{U}$ (see Figure 1). This condition is stronger than $\Pi_{p, \mathbf{q}}$ being transversal to $M_{U}$.

Let $\eta>0$ be arbitrary and let $N \in \mathbb{N}$ be sufficiently large. By Minkowski's linear forms theorem, given a point $u \in U$, there exist $\mathbf{q}=\mathbf{q}(u)=\left(q_{1}, \ldots, q_{n}\right) \in \mathbb{Z}^{n}$ satisfying $1 \leqslant|\mathbf{q}| \leqslant N$, and $p=p(u) \in \mathbb{Z}$ such that

$$
\left\{\begin{array}{l}
|\mathbf{q} \cdot \theta(u)-p| \leqslant N^{-n+k \eta}(\log N)^{k} \\
\left|q_{i}\right| \leqslant N, \quad i=1, \ldots, m \\
\left|q_{m+j}\right| \leqslant N^{1-\eta}(\log N)^{-1}, \quad j=1, \ldots, k
\end{array}\right.
$$

Hence for each $N=1,2, \ldots$, the set $U$ can be written

$$
U=A(N) \cup S(N) \cup E(N),
$$

where $E(N)=\{u \in U: \operatorname{dist}(u, \partial U) \leqslant 1 / N\}(\partial U$ is the boundary of $U)$,

$$
S(N)=\left\{u \in U: 1 \leqslant|\mathbf{q}|<N^{1-\eta} \text { for some q satisfying (7) }\right\},
$$

and

$$
A(N)=U \backslash(E(N) \cup S(N)),
$$

so that $A(N)$ consists of points $u \in U \backslash E(N)$ for which there exist $\mathbf{q} \in \mathbb{Z}^{n}$ and $p \in \mathbb{Z}$ satisfying (7) and

$$
N^{1-\eta} \leqslant|\mathbf{q}| \leqslant N
$$


Thus each $u \in A(N)$ is at least $1 / N$ from $\partial U$ (in the supremum metric), and there exists a large vector $\mathbf{q} \in \mathbb{Z}^{n}$ and an integer $p$ satisfying (7).

The measure of $E(N)$ converges to 0 as $N \rightarrow \infty$ since

$$
|E(N)|=\left|\left\{u \in U: \operatorname{dist}(u, \partial U) \leqslant \frac{1}{N}\right\}\right| \ll \ell(U)^{m}-\left(\ell(U)-\frac{1}{N}\right)^{m} \ll N^{-1} .
$$

The vector $\mathbf{q}=\mathbf{q}(u) \in \mathbb{Z}^{n}$ can be written

$$
\mathbf{q}=\left(q_{1}, \ldots, q_{m}, 0, \ldots, 0\right)+\left(0, \ldots, 0, q_{m+1}, \ldots, q_{n}\right)=\mathbf{q}^{\prime}+\mathbf{q}^{\prime \prime},
$$

say, where $\mathbf{q}^{\prime} \in \mathbb{R}^{m} \times\{(0, \ldots, 0)\}$ and $\mathbf{q}^{\prime \prime} \in\{(0, \ldots, 0)\} \times \mathbb{R}^{k}$. Since $N$ is large enough, for each $u \in A(N)$, the vector $\mathbf{q}$ is close to being parallel to $\mathbf{q}^{\prime}$. Indeed the angle $\beta$ that $\mathbf{q}$ makes with $\mathbb{R}^{m} \times\{0\}$ satisfies

$$
\cos \beta=\frac{\mathbf{q}}{|\mathbf{q}|_{2}} \cdot \frac{\mathbf{q}^{\prime}}{\left|\mathbf{q}^{\prime}\right|_{2}} \geqslant 1-\frac{q_{m+1}^{2}+\cdots+q_{n}^{2}}{|\mathbf{q}|_{2}^{2}}=1-O\left(\frac{1}{\log N}\right)^{2}
$$

by (7) and (9). Hence the hyperplane $\Pi_{p, \mathbf{q}}$, which is normal to $\mathbf{q}$, meets $M_{U}$ not close to tangentially. This implies that $\Pi_{p, \mathbf{q}} \cap M_{U}$ is a connected $(m-1)$-dimensional submanifold of $M_{U}$.

On replacing $N$ by $N^{1 /(1-\eta)}$ in (7), it can be seen that the set $S\left(N^{1 /(1-\eta)}\right)$ is contained in the set of points $u \in U$ for which there exist $p$, q satisfying

$$
|\mathbf{q} \cdot \theta(u)-p|<N^{-(n-k \eta) /(1-\eta)}(\log N)^{k}(1-\eta)^{-k}
$$

with $1 \leqslant|\mathbf{q}| \leqslant N$. Moreover, $S\left(N^{1 /(1-\eta)}\right)$ is also a subset of

$$
T_{\delta}(N)=\left\{u \in U:|\mathbf{q} \cdot \theta(u)-p|<N^{-n-\delta} \text { for some } \mathbf{q} \in \mathbb{Z}^{n}, p \in \mathbb{Z}, 1 \leqslant|\mathbf{q}| \leqslant N\right\},
$$

where $0<\delta<\eta(n-k) /(1-\eta)$.

Lemma 1. For any $\delta>0$,

$$
\limsup _{N \rightarrow \infty} T_{\delta}(N)=\bigcap_{k=1}^{\infty} \bigcup_{N=k}^{\infty} T_{\delta}(N) \subseteq L_{n+\delta} .
$$

Proof. Let $u \in \cap_{k=1}^{\infty} \cup_{N=k}^{\infty} T_{\delta}(N)$. Then $u \in T_{\delta}\left(N_{j}\right)$ for an infinite subsequence $N_{j}, j=1,2, \ldots$. Hence for each $j$ there exist $\mathbf{q}^{(j)} \in \mathbb{Z}^{n}$ with $1 \leqslant\left|\mathbf{q}^{(j)}\right| \leqslant N_{j}$ and $p^{(j)} \in \mathbb{Z}$ such that

$$
\left|\mathbf{q}^{(j)} \cdot \theta(u)-p^{(j)}\right|<N_{j}^{-n-\delta}
$$


Suppose there are only finitely many different $\mathbf{q}^{(j)}$ for which the last displayed inequality holds and let

$$
\min \left\{\left|\mathbf{q}^{(j)} \cdot \theta(u)-p^{(j)}\right|: j \in \mathbb{N}\right\}=c,
$$

say. If $c>0$, then choosing $j$ so that $N_{j}^{-n-\delta}<c$ gives a contradiction. If $c=0$, then for each $r \in \mathbb{N}, r \leqslant\left|r \mathbf{q}^{(j)}\right| \leqslant r N_{j}$ and

$$
\left|\left(r \mathbf{q}^{(j)}\right) \cdot \theta(u)-\left(r p^{(j)}\right)\right|=0<\left(r N_{j}\right)^{-n-\delta}
$$

Thus there are infinitely many solutions, contradicting the supposition that there exist only a finite number of different $\mathbf{q}^{(j)}$. But $1 \leqslant\left|\mathbf{q}^{(j)}\right| \leqslant N_{j}$, whence

$$
\left|\mathbf{q}^{(j)} \cdot \theta(u)-p^{(j)}\right|<\left|\mathbf{q}^{(j)}\right|^{-n-\delta}
$$

holds for infinitely many $j$. Thus $u \in L_{n+\delta}$.

By Fatou's lemma, for any $\delta>0$,

$$
\limsup _{N \rightarrow \infty}\left|T_{\delta}(N)\right| \leqslant\left|\limsup _{N \rightarrow \infty} T_{\delta}(N)\right| \leqslant\left|L_{n+\delta}\right|=0
$$

since $M$ is extremal. Thus $\lim _{N \rightarrow \infty}\left|T_{\delta}(N)\right|=0$. But when $0<\delta<\eta(n-k) /(1-\eta)$, $T_{\delta}(N) \supseteq S\left(N^{1 /(1-\eta)}\right)$, and so

$$
\lim _{N \rightarrow \infty}|S(N)|=\lim _{N \rightarrow \infty}\left|S\left(N^{1 /(1-\eta)}\right)\right|=0 .
$$

Applying this and the estimate for $|E(N)|$ above to (8), it follows that

$$
|U \backslash A(N)| \leqslant|E(N)|+|S(N)| \longrightarrow 0
$$

as $N \rightarrow \infty$ and $A(N)$ satisfies (3). The resonant sets in $U$ are now chosen to be

$$
R_{p, \mathbf{q}}=\{u \in U: \mathbf{q} \cdot \theta(u)=p\}=h\left(\Pi_{p, \mathbf{q}} \cap M_{U}\right),
$$

where $\mathbf{q}$ and $p$ are given by (7). Thus $d$, the dimension of $R_{p, \mathbf{q}}$, is $m-1$.

For each $u \in A(N)$, there exists a pair $(p, \mathbf{q})$ satisfying (7) and $N^{1-\eta} \leqslant|\mathbf{q}| \leqslant N$. For $N$ sufficiently large, the hyperplane $\Pi_{p, \mathbf{q}}$ is far from tangential to $M_{U}$. Because of this and $\theta$ being bi-Lipschitz,

$$
\operatorname{dist}\left(u, R_{p, \mathbf{q}}\right) \asymp \operatorname{dist}\left(\theta(u), \theta\left(R_{p, \mathbf{q}}\right)\right) \asymp \frac{|\mathbf{q} \cdot \theta(u)-p|}{|\mathbf{q}| 2|\cos \varpi|},
$$

where $\varpi$ is the angle between the tangent plane $T_{\theta(u)} M_{U}$ and $\mathbf{q}$. Since $\Pi_{p, \mathbf{q}}$ meets $M_{U}$ not close to tangentially, $\cos \varpi \asymp 1$, and so for any $u \in U$,

$$
\operatorname{dist}\left(u, R_{p, \mathbf{q}}\right) \asymp \frac{|\mathbf{q} \cdot \theta(u)-p|}{|\mathbf{q}|_{2}} .
$$


It follows from this and (7) that there are positive $c_{*}, c^{*}$ such that

$$
c_{*}|\mathbf{q} \cdot \theta(u)-p||\mathbf{q}|^{-1} \leqslant \operatorname{dist}\left(u, R_{p, \mathbf{q}}\right) \leqslant c^{*} N^{-n-1+\eta(k+1)}(\log N)^{k} .
$$

Let

$$
\rho(N)=4 c^{*} N^{-n-1+(k+1) \eta}(\log N)^{k} .
$$

We now show that the other ubiquity properties (5) and (6) hold for the family $\mathscr{R}$ of resonant sets $\left\{R_{p, \mathbf{q}}\right\}$ where $\lfloor(p, \mathbf{q})\rfloor=|\mathbf{q}|$ and $\rho: \mathbb{N} \rightarrow \mathbb{R}^{+}$is given by (13). Let $H$ be a hypercube with $\ell(H)=\rho(N)$. The choice of $\mathbf{q}$, which ensures that $\Pi_{p, \mathbf{q}}$ meets $M_{U}$ not close to tangentially, together with the choice of $\rho$, implies that if in addition $u \in H / 4$, then by (12), there exist $p$, q such that $\operatorname{dist}\left(u, R_{p, \mathbf{q}}\right) \leqslant \ell(H) / 4$. Hence the resonant set $R_{p, \mathbf{q}}$ meets the hypercube $H$ substantially and

$$
\left|H \cap B\left(R_{p, \mathbf{q}} ; \delta\right)\right| \gg \ell(H)^{m-1} \delta,
$$

where $B\left(R_{p, \mathbf{q}} ; \delta\right)$ is given by (4), as required for (5) to hold.

It also follows that $\Pi_{p, \mathbf{q}}$ meets $M_{U}$ in a connected $(m-1)$-dimensional submanifold, so that any hypercube $H^{\prime}$ with $\ell\left(H^{\prime}\right) \leqslant \rho(N)$ satisfies

$$
\left|H^{\prime} \cap H \cap B\left(R_{p, \mathbf{q}} ; \delta\right)\right| \ll \ell\left(H^{\prime}\right)^{m-1} \min \left\{\delta, \ell\left(H^{\prime}\right)\right\} \ll \ell\left(H^{\prime}\right)^{m-1} \delta,
$$

and (6) holds. Thus the family $\mathscr{R}=\left\{R_{p, \mathbf{q}}: \mathbf{q} \in \mathbb{Z}^{n} \backslash\{0\}, p \in \mathbb{Z}\right\}$ is ubiquitous in $U$ with respect to $\rho$. Hence by Theorem 1 , for any decreasing function $\widetilde{\psi}: \mathbb{N} \rightarrow \mathbb{R}^{+}$,

$$
\operatorname{dim} \Lambda(\mathscr{R} ; \widetilde{\psi}) \geqslant m-1+\gamma
$$

where $\Lambda(\mathscr{R} ; \widetilde{\psi})$ is the set of points $u$ in $U$ satisfying

$$
\operatorname{dist}\left(u, R_{p, \mathbf{q}}\right)<\widetilde{\psi}(\lfloor(p, \mathbf{q})\rfloor)=\widetilde{\psi}(|\mathbf{q}|)
$$

for infinitely many $p, \mathbf{q}$ and where $\gamma=\limsup \operatorname{sum}_{N \rightarrow \infty}(\log \rho(N)) /(\log \widetilde{\psi}(N))$.

Choose $\widetilde{\psi}(r)=c_{*} r^{-1} \psi(r)$. Then by (12), $\operatorname{dist}\left(u, R_{p, \mathbf{q}}\right)<\widetilde{\psi}(|\mathbf{q}|)$ implies that $|\mathbf{q} \cdot \theta(u)-p|<\psi(|\mathbf{q}|)$. Therefore $u \in \Lambda(\mathscr{R} ; \widetilde{\psi})$ implies that for infinitely many $p, \mathbf{q}$,

$$
|\mathbf{q} \cdot \theta(u)-p|<\psi(|\mathbf{q}|),
$$

and so $\Lambda(\mathscr{R} ; \widetilde{\psi}) \subset L(\psi)$. Thus

$$
\operatorname{dim} L(\psi) \geqslant \operatorname{dim} \Lambda(\Re ; \widetilde{\psi}) \geqslant m-1+\gamma,
$$

where by (13)

$$
\gamma=\limsup _{N \rightarrow \infty} \frac{\log \rho(N)}{\log \left(c_{*} N^{-1} \psi(N)\right)}=\frac{n+1-\eta(k+1)}{\lambda+1},
$$


where $\lambda$ is the lower order of $1 / \psi$. Since $\eta$ is an arbitrary positive number and $U$ is a parametrisation domain, it follows that

$$
\operatorname{dim} \mathscr{L}(M ; \psi) \geqslant \operatorname{dim} L(\psi) \geqslant m-1+\frac{n+1}{\lambda+1},
$$

and Theorem 2 is proved.

By [11], a $C^{r} m$-dimensional manifold embedded in $\mathbb{R}^{n}$ and $\ell$-nondegenerate for some $\ell \leq r$ almost everywhere is extremal $\left(\theta(U)\right.$ is $\ell$-nondegenerate if $\mathbb{R}^{n}$ is spanned by the partial derivatives of $\theta$ up to order $\ell$ ). Hence (14) holds for such manifolds with $r \geq 1$ and so in particular for manifolds with at least one principal curvature nonzero almost everywhere. If $M$ is not extremal, then $\operatorname{dim} \mathscr{L}_{w}(M)=m$ for some $w>n$, and hence $\operatorname{dim} \mathscr{L}_{v}(M)=m$ for $v \leqslant w$.

Obtaining an upper bound for the Hausdorff dimension of $\mathscr{L}(M ; \psi)$ involves estimating large contributions from near tangential resonant sets $R_{p, \mathbf{q}}$ and is much more difficult. The upper bound for $\mathscr{L}(M ; \psi)$ has been shown to be $m-1+(n+1) /(v+1)$ for $v \geqslant n$ when $M$ is $C^{3}$, of dimension $m \geqslant 2$, and has at least two principal curvatures nonzero everywhere except on a set of Hausdorff dimension at most $m-1$ (see [7]), so that the lower bound in Theorem 2 is best possible. It is likely that this is the Hausdorff dimension when at least one principal curvature is nonzero everywhere except on a set of Hausdorff dimension at most $m-1$. Determining the Hausdorff dimension in the case of simultaneous Diophantine approximation seems harder and much less is known.

\section{REFERENCES}

[1] A. Baker, Transcendental Number Theory, Cambridge Univ. Press, London, 1975.

[2] A. BAKER AND W. M. Schmidt, Diophantine approximation and Hausdorff dimension, Proc. London Math. Soc. (3) 21 (1970), 1-11.

[3] R. C. BAKER, Dirichlet's theorem on Diophantine approximation, Math. Proc. Cambridge Philos. Soc. 83 (1978), 37-59.

[4] V. Beresnevich And V. Bernik, On a metrical theorem of W. Schmidt, Acta Arith. 75 (1996), 219-233.

[5] V. I. BERNIK, Application of the Hausdorff dimension in the theory of Diophantine approximations (in Russian), Acta Arith. 42 (1983), 219-253.

[6] - A proof of Baker's conjecture in the metric theory of transcendental numbers (in Russian), Dokl. Akad. Nauk SSSR 277 (1984), 1036-1039.

[7] M. M. Dodson, B. P. Rynne, And J. A. G. Vickers, Metric Diophantine approximation and Hausdorff dimension on manifolds, Math. Proc. Cambridge Philos. Soc. 105 (1989), 547-558.

[8] - Diophantine approximation and a lower bound for Hausdorff dimension, Mathematika 37 (1990), 59-73.

[9] - Khintchine-type theorems on manifolds, Acta Arith. 57 (1991), 115-130.

[10] K. J. Falconer, The Geometry of Fractal Sets, Cambridge Tracts in Math. 85, Cambridge Univ. Press, Cambridge, 1986.

[11] D. Y. Kleinbock AND G. A. Margulis, Flows on homogeneous spaces and Diophantine approximation on manifolds, Ann. of Math. (2) 148 (1998), 339-360. 
[12] J. F. Koкsma, Diophantische Approximationen, Springer-Verlag, Berlin, 1974.

[13] J. KubILIUS, On an application of I. M. Vinogradov's method to the solution of a problem of the metrical theory of numbers (in Russian), Dokl. Akad. Nauk SSSR 67 (1949), 783-786.

[14] P. MatriLa, Geometry of Sets and Measures in Euclidean Spaces: Fractals and Rectifiability, Cambridge Stud. Adv. Math. 44, Cambridge Univ. Press, Cambridge, 1995.

[15] B. P. RYNNE, Regular and ubiquitous systems, and $\mathcal{M}_{\infty}^{s}$-dense sequences, Mathematika 39 (1992), 234-243.

[16] W. M. SchмIDT, Metrische Sätze über simultane Approximation abhängiger Grössen, Monatsh. Math. 68 (1964), 154-166.

[17] V. G. SprindžUK, Mahler's Problem in Metric Number Theory, Transl. Math. Monogr. 25, Amer. Math. Soc., Providence, 1969.

[18] - Metric Theory of Diophantine Approximations, Scripta Ser. Math., Wiley, New York, 1979.

[19] - Achievements and problems of the theory of Diophantine approximations (in Russian), Uspekhi Mat. Nauk 35 (1980), 3-68, 248; English translation in Russian Math. Surveys 35 (1980), 1-80.

[20] A. I. Vinogradov and G. V. Chudnovsky, "The proof of extremality of certain manifolds" in Contributions to the Theory of Transcendental Numbers, Math. Surveys Monogr. 19, Amer. Math. Soc., Providence, 1984, 421-447.

Department of Mathematics, University of York, York YO1 5DD, England; hd3@ york.ac. uk; mmd1@york.ac.uk 